\title{
Optimización de parámetros para modulación Nyquist óptica y comparación con OFDM óptica
}

\author{
José A. Altabás ${ }^{1}$, José A. Lázaro ${ }^{2}$, Félix Sotelo ${ }^{1}$, Ignacio Garcés ${ }^{1}$ \\ ${ }^{1}$ Grupo de Tecnologías Fotónicas, \\ Instituto de Investigación en Ingeniería de Aragón (I3A). \\ Universidad de Zaragoza, Mariano Esquillor s/n, 50018, Zaragoza, Spain. \\ Tel. +34-976762707, Fax +34-976762043, e-mail: jaltabas@unizar.es \\ ${ }^{2}$ Universidad Politécnica de Cataluña
}

\begin{abstract}
En este artículo se presenta un análisis y optimización de parámetros de modulación Nyquist óptica y su comparación con Orthogonal FrequencyDivision Multiplexing (OFDM) óptica. Este análisis se ha centrado en un esquema de modulación en intensidad y detección directa para su extensión en sistemas de mayor rentabilidad.
\end{abstract}

\section{Introducción}

En los últimos años, se ha producido un crecimiento exponencial del tráfico de datos en Internet. Este crecimiento ha motivado el desarrollo de nuevas redes ópticas que permitan afrontar esta demanda, haciendo un uso eficiente de los recursos de las redes ópticas mediante Elastic Optical Networks (EON) [1], [2]. Estas redes realizan una asignación adaptativa del espectro óptico en función de la demanda de tráfico, la disponibilidad dinámica de los recursos, etc.

En este artículo presentamos el análisis y optimización de la modulación Nyquist digital, en función de los requerimientos de ancho de banda eléctrico del receptor, relación señal a ruido óptica (OSNR) y de la longitud del pulso conformador. Además, se realiza un análisis comparativo de la modulación Nyquist digital con OFDM como referencia. Esta comparativa se centra en modulación en intensidad y detección directa para su potencial extensión a sistemas mas rentbles tales como RSOAs o láseres modulados directamente [3].

\section{Setup}

La simulación presentada en este artículo se realiza mediante el esquemático mostrado en la Figura 1 utilizando Matlab ${ }^{\mathrm{TM}}$ y VPItransmissionMaker ${ }^{\mathrm{TM}}$

El bloque “Generador de Símbolos/DAC" se generan los datos mediante Matlab ${ }^{\mathrm{TM}}$ y realiza la modulación deseada. En la modulación Nyquist, los datos son filtrados mediante un conformador de pulso (función sinc), el cual tiene una longitud infinita por lo que debe ser truncado. OFDM se obtiene mediante la IFFT de un grupo de 128 subportadoras, que debe tener simetría hermítica, es decir, debe tener la siguiente forma: $\left[\begin{array}{llllllll}0 & a_{1} & \ldots & a_{63} & 0 & a_{63} & \ldots & a_{1}\end{array}\right]$ donde $a_{i}$ representa el dato transmitido en cada subportadora, para evitar producir señales complejas. En este bloque se selecciona la tasa de muestreo del DAC, que será la misma que la del ADC en "Muestreador/ADC".

“Bias-Tee y Driver” amplifican la señal y añaden el nivel de continua necesario para que la señal sea puramente positiva y pueda modular en intensidad, mediante "Modulador Mach-Zehnder", a la luz emitida por el bloque "Laser". La señal óptica generada pasa a través del bloque "Set OSNR" que añade ruido ASE para obtener una OSNR contralada y después es demodulada con un fotodiodo (“PIN”).

En “Adquisición y postprocesado", la señal de modulación Nyquist es demodulada mediante un decisor de umbral, mientras que la señal de OFDM es procesada mediante FFT para recuperar los datos.

\section{Resultados}

\section{Longitud del pulso conformador de Nyquist}

El truncado en el pulso conformador de Nyquist tiene un efecto minimo sobre la evolucón de la tasa de error en función del ancho de banda del receptor, tal y como se puede observar en la Figura 2. El principal efecto de estas variaciones aparece en el espectro óptico de la señal. En la Figura 3, se observa como se produce un ensanchamiento progresivo del espectro conforme se reduce el numero de simbolos de longitud del pulso conformador. Además, cuando esta longitud es pequeña aparecen unas replicas de la portadora que no son filtradas correctamente por la modificación de la respuesta espectral del filtro conformador. 


\section{Comparativa entre Nyquist y OFDM}

Observando la Figura 4 se observa que la modulación Nyquist se comporta mejor que la OFDM en casi un orden de magnitud. Esto se debe a los efectos de propagación de errores al recuperar los datos mediante la FFT en OFDM. La modulación Nyquist permite una reducción del 20\% de ancho de banda eléctrico del receptor respecto a OFDM para obtener la misma tasa de error.

Los espectros de ambas modulación son muy similares, tal y como se ve en la Figura 5. El espectro de Nyquist con un pulso conformado de 128 símbolos de longitud tiene una caída en el espectro mucho más abrupta entre la banda de interés y la adyacente que en el caso de OFDM

\section{Conclusiones}

En este artículo, se ha analizado la modulación Nyquist comparándola con la modulación OFDM. La longitud del pulso conformador de la modulación Nyquist afecta a principalmente a la forma del espectro dejando prácticamente igual la tasa de error. El compromiso entre el coste computacional y la forma espectral es la utilización de un pulso conformador de longitud 12 símbolos. Además, se ha observado que el uso de una modulación Nyquist

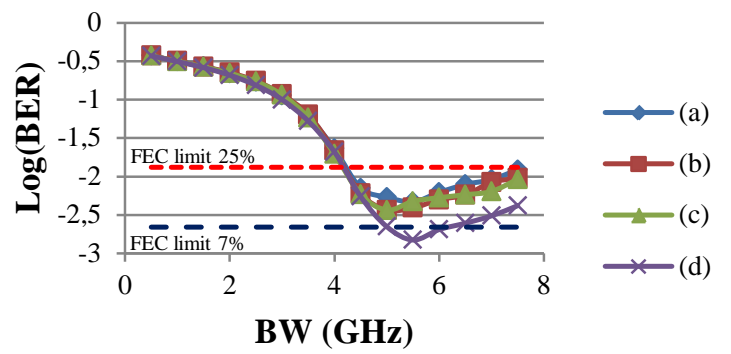

Figura 2. BER vs. Ancho de banda eléctrico del receptor (BW) para conformador de pulso Nyquist con 6 muestras por símbolo a $10 \mathrm{~Gb} / \mathrm{s}$, OSNR=15dB y pulso conformador truncado a: (a) 128, (b) 32, (c) 12 y (d) 4 símbolos.

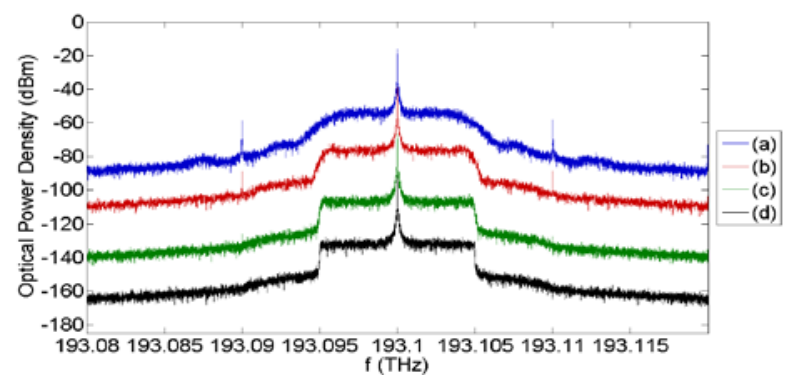

Figura 3. Espectro óptico de Nyquist digital a 10Gb/s con 6 muestras por símbolo y pulso conformador truncado a: (a) 4, (b) 12, (c) 32, (d) 128 símbolos de longitud. Los espectros (b-d) sean reescalado $25 \mathrm{~dB}$ para mejorar su visibilidad. permite utilizar receptores con un ancho de banda eléctrico del $80 \%$ de ancho de banda de la señal obteniendo la misma tasa de error que en OFDM.

\section{REFERENCIAS}

[1]. GERSTEL, O., JINNO, M., LORD, A. and YOO, S.J.B. Elastic Optical Networking: A New Draw for the Optical Layer? IEEE Communications Magazine. 2012, 50(2), 12-20.

[2]. JINNO, M., KOZICKI, B., TAKARA, H., WATANABE, A., SONE, Y., TAKANA, T., and HIRAN, A. Distance-Adaptative Spectrum Resource Allocation In Spectrum-sliced Elastic Optical Path Network. IEEE Communications Magazine. 2010, 48(8), 138-145.

[3]. LAZARO, J.A., KNORR, S., SCHRENK, B., CANO, I., POLO, V., PRAT, J. CARENA, A. and BOSCO,

G. Digital Nyquist WDM for Access Networks using Limited Bandwidth Reflective Semiconductor Optical Amplifiers. In: Proc. Of OFC/NFOEC 2012. [Los Angeles]: IEEE, 2012, pp. 1-3.

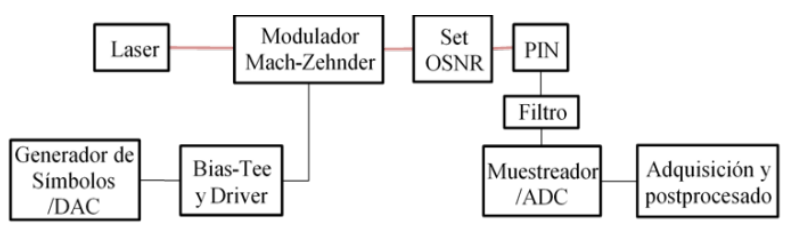

Figura 1. Setup de simulación (VPItransmissionMaker ${ }^{\mathrm{TM}}$ )

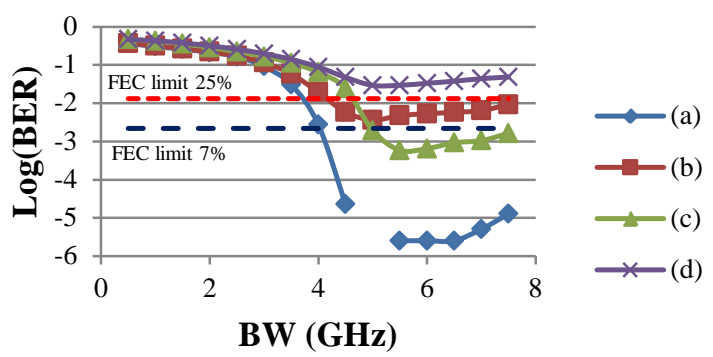

Figura 4. BER vs. Ancho de banda eléctrico del receptor (BW) para conformador de pulso Nyquist truncado a 12 símbolos y OFDM ideal, ambos con 6 muestras por símbolo a 10Gb/s. (a) y (b) modulación Nyquist, , (c) y (d) OFDM. (a) y (c) OSNR=20dB, (b) y (d) OSNR=15dB.

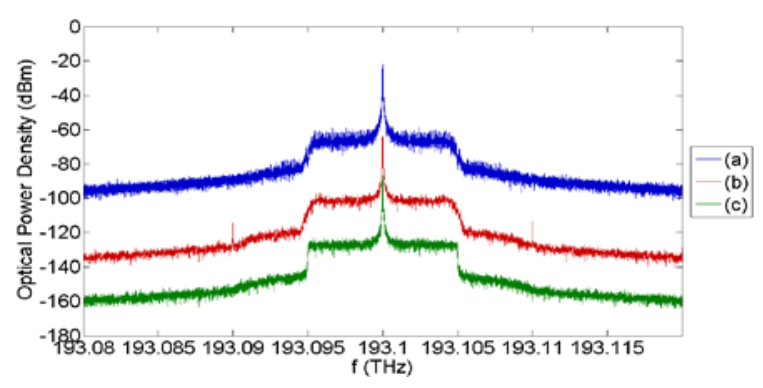

Figura 5. Espectros ópticos de Nyquist digital y OFDM a 10Gb/s con 6 muestras por símbolo. (a) OFDM, (b) y (c) Nyquist con 12 y 128 símbolos de longitud de pulso conformador. (b) ha sido reescalado $50 \mathrm{~dB}$ y (c) $30 \mathrm{~dB}$ mas. 